\title{
Duration of components and response rates on multiple fixed-ratio schedules
}

\author{
JIM NORBORG, STEVE OSBORNE, and EDMUND FANTINO \\ University of California, San Diego, La Jolla, California
}

\begin{abstract}
Pigeons were exposed to a two-component multiple fixed-ratio $X$ fixed-ratio $Y$ schedule of reinforcement in which $X$ was always less than $Y$. Components were equal in duration and alternated at rates that varied between $2 \mathrm{sec}$ and $23.5 \mathrm{~h}$. Relative response rate in the FR X component: (1) increased as the duration of components increased between $2 \mathrm{sec}$ and $15 \mathrm{~min}$, (2) was at a maximum between $15 \mathrm{~min}$ and $6 \mathrm{~h}$, and (3) decreased as the duration of components increased from $6 \mathrm{~h}$ to $23.5 \mathrm{~h}$. The changes in relative response rate were attributable primarily to changes in absolute response rates during the FR Y schedule as absolute response rates during the FR $X$ schedule were relatively invariant. These results pose complexities for several theoretical formulations.
\end{abstract}

In a multiple (mult) schedule, two or more independent reinforcement schedules are alternated and each of the component schedules is accompanied by a different stimulus. Although the four most common simple schedules, fixed interval (FI), fixed ratio (FR), variable interval (VI), and variable ratio (VR), have all been studied as components of mult schedules, VI schedules have received the most attention. The comparison of different schedule combinations, although important in its own right, is also relevant to several attempts to integrate data from a wide range of experimental procedures (Catania, 1973; Herrnstein, 1970, 1979; Rachlin, 1973; Shimp, 1969, 1973). Most of the data cited by these investigators, in support of their respective positions, come from experiments employing interval, rather than ratio, schedules.

Data from ratio schedules would appear to permit an assessment of the power and generality of these formulations. It is easy to see, however, why data from mult or concurrent (conc) ratio schedules are seldom included in these analyses. In the first place, the few studies that have examined responding on mult FR FR schedules may not be comparable to those involving mult VI VI schedules. For example, the transition from one component to the next in a mult VI VI has been response independent (Lander \& Irwin, 1968; Nevin \& Shettleworth, 1966; Reynolds, 1963; Shimp \& Wheatley, 1971; Todorov, 1972); that is, the components of a two-component mult schedule alternate at some specified interval, independently of the animal's behavior. With mult

This research was supported by NIMH Grant 20752 to the University of California, San Diego. We thank Mary Thompson for unfailing assistance. Reprints may be obtained from Edmund Fantino, Department of Psychology, C-009, University of California, San Diego, La Jolla, California 92093.
FR FR, however, the transition has been response dependent, since the components typically alternate after some specified number of reinforcements (Crossman, 1968, 1971; Findley, 1962; Schuster, 1959). More importantly, the relation between responding and reinforcement rate, of central interest on interval schedules, is not free to vary on ratio schedules. However, experiments by Shimp and Wheatley (1971) and Todorov (1972) with mult VI VI schedules suggest a promising procedure for studying behavior maintained by mult FR FR reinforcement schedules and for meaningfully comparing such behavior with that reported in the mult VI VI case. These studies examined the rates of responding on a mult VI VI schedule as a function of the duration of the mult schedule components. In each case, the relative response rate (the response rate in one component divided by the sum of the response rates in both components) in the component associated with the higher reinforcement rate increased as the duration of the components decreased. Moreover, with sufficiently short component durations, relative response rates on a mult VI VI schedule equaled or "matched" the relative rates of reinforcement obtained on that schedule as they often do on conc VI VI schedules (e.g., Herrnstein, 1970). These findings are consistent with the formulations of both Herrnstein (1970) and Rachlin (1973), who provided the most explicit treatment of mult-schedule behavior.

The present series of experiments is analogous to those of Shimp and Wheatley (1971) and Todorov (1972) insofar as they also study the effects of component duration on the relative and absolute rates of responding in mult schedules. In the present work, however, responding on mult FR FR schedules is investigated. In particular, we examine the effects of both component and session duration on mult 
FR FR schedules (in which transitions between components are response independent, as they typically are in mult VI VI schedules).

The importance of session duration for responding on simple schedules of reinforcement has been stressed by Hursh (1980). He reviewed several studies that suggest that response rates are a function of session length. Hursh argues persuasively that the different sets of results depend upon the control of food consumption both inside and outside the experimental chamber. When subjects are studied $24 \mathrm{~h}$ per day, all their food is earned in the chamber (a closed economy). Thus, as the schedule provides less and less food per time or per response, response rates must increase to maintain an adequate level of consumption. When food is freely provided after a relatively short experimental session, however, the relation between the subject's performance in the experiment and its level of consumption is less constrained (a relatively open economy). Here rate of responding need not-and apparently does not-increase as decreasing amount of food is provided per time or response. It is likely that in multiple schedules component duration might also affect subjects' responding, especially with ratio schedules. Responding is likely to be more equally maintained on both long and short ratios when component duration exceeds the subjects' normal intermeal interval. The expected relation between component duration and responding is less clear, however, for shorter durations. On the one hand, the subject might pause throughout the component with the larger ratio until the shorter ratio was reinstated, thus minimizing response output. On the other hand, if the components were so short that a meal initiated in the smaller ratio component could not be completed, then the subject might respond in both components, thus preserving a preferred pattern of food intake. The present experiments examined relative and absolute rates of responding on multiple ratio schedules as component duration varied.

\section{EXPERIMENT 1}

A first concern in evaluating the effect of component duration on response rate in mult FR FR schedules was the possibility that session duration, rather than component duration, might completely determine response allocation to the two schedules. This concern stemmed from the fact that as session duration is increased there is an increase in the total time that both components are available and hence an opportunity for the pigeon to gain a greater amount of reinforcement from the component associated with the smaller response requirement. The purpose of Experiment 1 was to determine whether an exclusive preference for the schedule associated with the smaller response requirement could be pro- duced simply by increasing session duration on a mult FR FR schedule.

\section{Method}

Subjects. Two adult male White Carneaux pigeons were maintained only by the reinforcements delivered during the experimental sessions. Both pigeons had been employed in previous experiments.

Apparatus. The experimental chamber (Ferster \& Skinner, 1957) contained two translucent response keys mounted $12.5 \mathrm{~cm}$ apart and $22 \mathrm{~cm}$ above the floor. The right key was dark and inoperative throughout the experiment. The left key could be transilluminated by either a white or green light. A minimum force of $0.1 \mathrm{~N}$ was required to operate the response key. The food magazine was equidistant from the two response keys and was $7 \mathrm{~cm}$ above the floor. The chamber was illuminated by a houselight except during reinforcement, when the houselight and key light went off and the magazine was illuminated. Reinforcement consisted of 4-sec access to mixed grain.

Procedure. The reinforcement schedule was mult FR 30 FR 90. The FR 30 schedule was associated with a green key light, and the FR 90 schedule, with a white key light; that is, in the presence of a green key light, the 30th response produced reinforcement, and in the presence of a white key light the 90th response produced reinforcement. When the completion of a ratio requirement was interrupted by a component change, the responses already made in that component counted toward completing the ratio requirement when the component in which they were made was once again available.

Component duration was either $1 \mathrm{~min}$ or $5 \mathrm{~min}$. In either case, the components alternated every $t \mathrm{~min}$ independently of the pigeon's behavior. Daily experimental sessions were terminated after 40,80 , or $160 \mathrm{~min}$. The subjects were exposed to eight conditions in the order listed in Table 1.

An experimental condition was in effect for a minimum of 15 daily sessions and until the relative frequency of reinforcement for nine consecutive sessions was stable. The relative frequency of reinforcement was calculated by taking the number of reinforcements obtained in the component associated with the smaller ratio requirement (FR 30) and dividing by the sum of the reinforcements obtained in both components. Stability was defined as follows: Starting with the seventh session, consecutive sessions were divided into blocks of three sessions each. When the mean relative frequency of reinforcement in three consecutive blocks $\left(\mathbf{M}_{1}, \mathbf{M}_{\mathbf{3}}\right.$, and $M_{3}$ ) deviated by no more than 0.05 from the grand mean $\left[\left(M_{1}+M_{2}+M_{3}\right)\right.$ divided by 3$]$ and no trend was observed (i.e., neither $\mathbf{M}_{1}>\mathbf{M}_{2}>\mathbf{M}_{3}$ nor $\mathbf{M}_{1}<\mathbf{M}_{2}<\mathbf{M}_{3}$ ), relative frequency of reinforcement was considered stable (on ratio schedules relative reinforcement ratio is a monotonic function of relative response rate, used more commonly to assess stability; relative reinforcement rate was used for convenience).

Table 1

The Sequence of Experimental Conditions and Number of Sessions for Experiment 1

\begin{tabular}{cccc}
$\begin{array}{c}\text { Component } \\
\text { Duration }\end{array}$ & $\begin{array}{c}\text { Session } \\
\text { Duration }\end{array}$ & \multicolumn{2}{c}{ Number of Sessions } \\
\cline { 3 - 4 } Subject 5 & Subject 14 \\
\hline 5 & 40 & 60 & 60 \\
1 & 40 & 45 & 45 \\
5 & 80 & 21 & 18 \\
5 & 80 & 27 & 27 \\
1 & 160 & 21 & 21 \\
1 & 160 & 30 & 36 \\
5 & 40 & 18 & 24 \\
\hline
\end{tabular}

Note-Durations are given in minutes. 


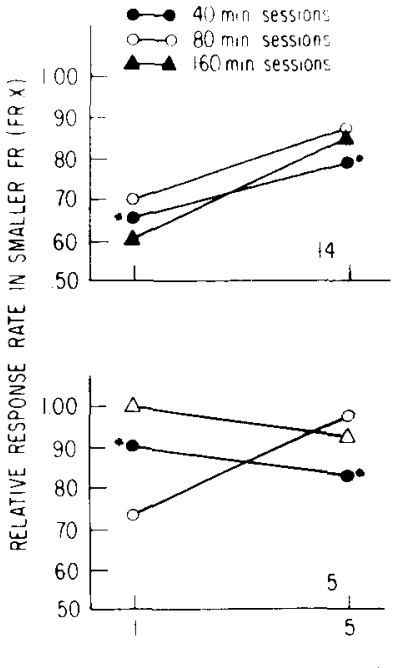

COMPONENT DURATION (MIN)
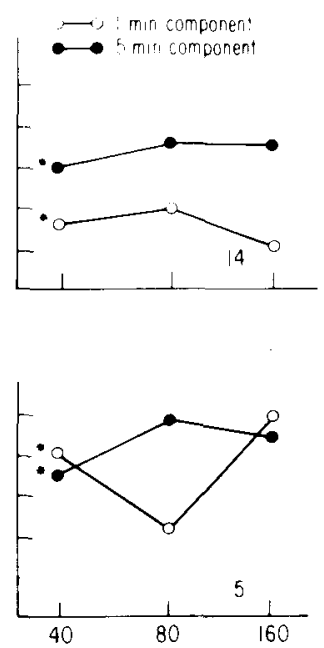

SESSION DURATION (MIN)

Figure 1. Relative response rate computed for smaller fixed ratio (FR X; $X=30$ ) as a function of component duration and sesslon duration in Experiment 1. Points marked with an asterisk (*) denote mesn of two observations.

\section{Results}

Response rates were calculated by dividing the number of responses emitted in the presence of a given schedule by the time spent in the presence of that schedule (reinforcement time excluded). Relative response rate was calculated by dividing the response rate in the presence of the green key light (FR 30) by the sum of the response rates in both components. Figure 1 shows relative response rate as a function of component duration and session duration. For Pigeon 14, relative response rate was consistently higher for 5-min components than for 1-min components. For Pigeon 5, there was no consistent relationship between component duration and relative

response rate. No systematic effect of session duration on relative response rate was observed for either pigeon.

\section{Discussion}

The results of Experiment 1 suggest two things. First, increasing session duration on a mult FR FR schedule is not sufficient to produce exclusive responding in the component associated with the lower response requirement. Second, the longer component duration did not result in lower relative response rates for either pigeon, and for Pigeon 14 relative response rate was consistently higher with the longer component duration, a finding opposite to that reported with mult VI VI schedules by Shimp and Wheatley (1971) and Todorov (1972).

\section{EXPERIMENT 2}

The purpose of Experiment 2 was to provide additional data on mult FR FR schedules by employing different ratio combinations and a greater range of component durations.

\section{Method}

Subjects. Four adult male White Carneaux pigeons were maintained only by the reinforcements delivered during the experimental sessions. All four pigeons had been employed in previous experiments.

Apparatus. The apparatus was the same as that described for Experiment 1.

Procedure. The reinforcement schedule was mult FR X FR Y $(X<Y)$. The $F R X$ schedule was associated with a green key light, and the FR $Y$ schedule, with a red key light. The values of $X$ and $Y$ for each pigeon are given below.

Component duration was varied between $2 \mathrm{sec}$ and $15 \mathrm{~min}$, and the session duration was 30,60 , or $90 \mathrm{~min}$. The sequence of experimental conditions' for each pigeon is given in the order shown in Table 2. All other procedural details were the same as those described for Experiment 1.

Table 2

The Sequence of Experimental Conditions and Number of Sessions in Experiment 2

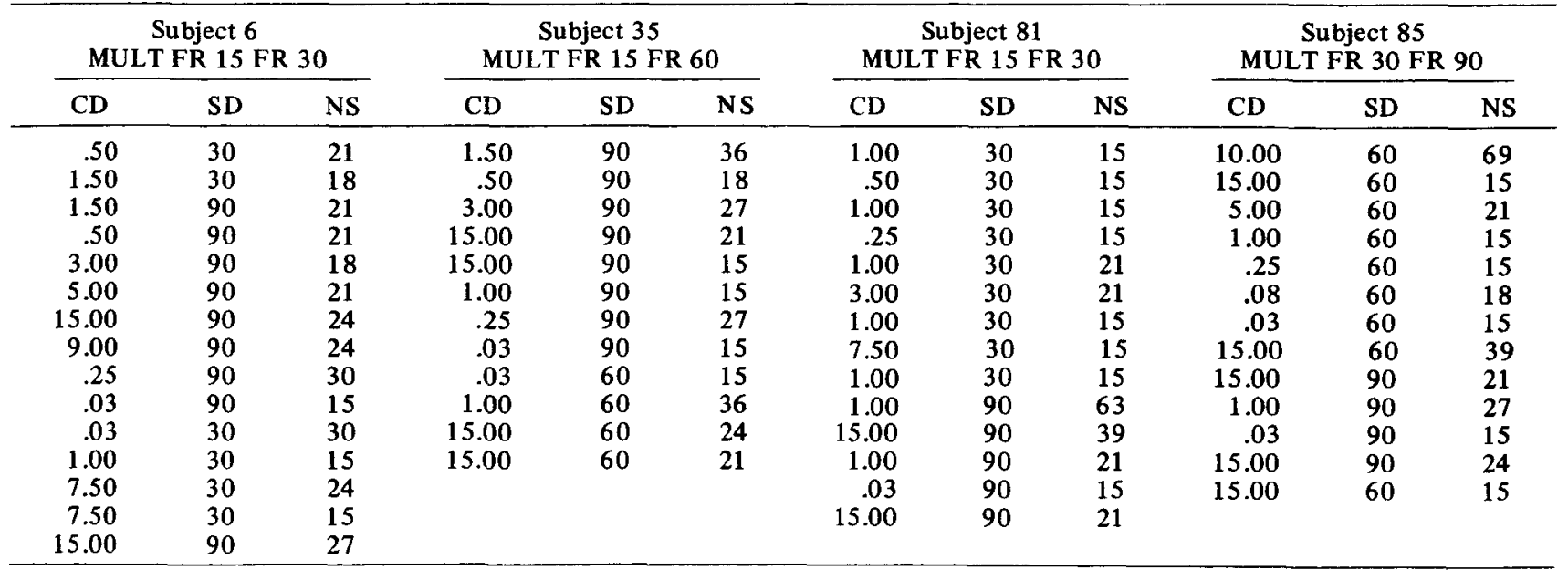

Note $-C D=$ component duration (in minutes); $S D=$ session duration (in minutes); $N S=$ number of sessions. 


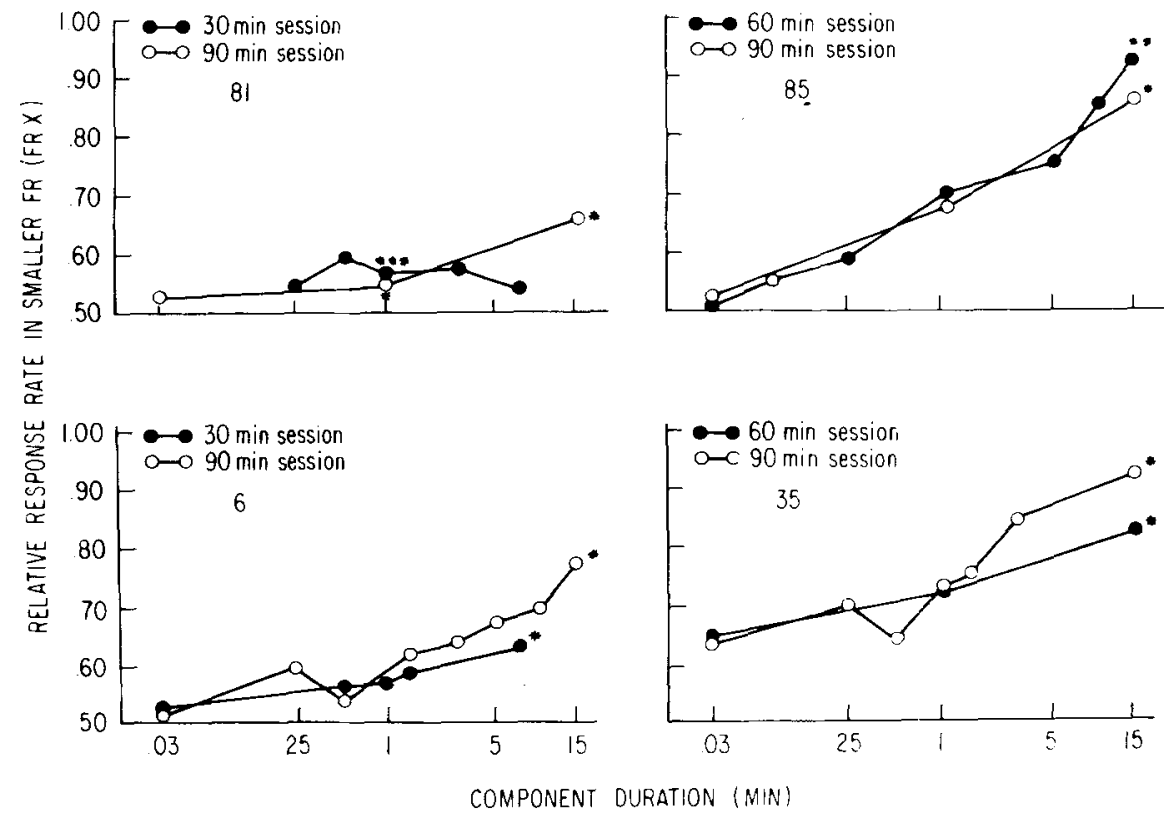

Figure 2. Relative response rate computed for smaller fixed ratio (FR $X$ ) as a function of component duration in Experiment 2. Points marked with one asterisk (*) denote mean of two observations. Two asterisks denote mean of three observations, and three asterisks denote mean of five observations.

\section{Results}

The values in Figures 2 and 3 are the means of data collected in nine consecutive sessions which met the stability criterion. Figure 2 shows relative response rate as a function of component duration for each pigeon. In every case except one (Pigeon 81 at a session duration of $30 \mathrm{~min}$ ), relative response rate increased as component duration increased. At the longer component durations, relative response rate was higher when the difference between the component schedules was large (i.e., Pigeon 85 , mult FR 30 FR 90; Pigeon 35, mult FR 15 FR 60) than when the difference was small (i.e., Pigeons 81 and 6, mult FR 15 FR 30).

Figure 3 shows absolute response rate as a function of component duration. Here, too, the results for Pigeon 81 are atypical, while the results for the other three pigeons are quite consistent. Component duration had little or no systematic effect on response rate in the component associated with the smaller response requirement (filled circles and triangles). Instead, as component duration increased, response rate in the component with the longer requirement decreased (open circles and triangles), producing the increase in relative response rate observed in Figure 2.

\section{Discussion}

On a mult FR FR schedule, relative response rate (in the component associated with the smaller re-

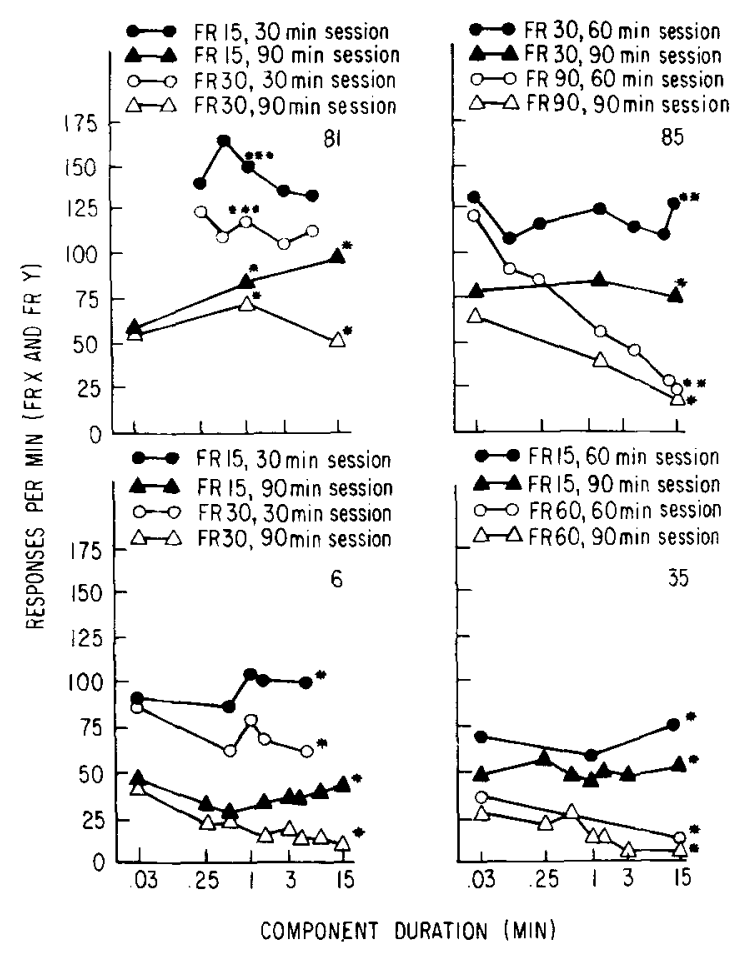

Figure 3. Responses per minute for smaller fixed ratlo (FR X) and larger flxed ratio (FR Y) as a function of component duration in Experiment 2. Points marked with one asterisk (") denote mean of two observations. Two asterisks denote mean of three observations, and three asterisks denote mean of five observations. 
sponse requirement) increases as component duration increases (in the range of $2 \mathrm{sec}$ to $15 \mathrm{~min}$ ). The results of Experiment 2 provide additional support for the more tentative assertion made in Experiment 1 , that the function relating relative response rate and component duration on mult FR FR schedules may be opposite to that found on mult VI VI schedules. The magnitude of this effect, in terms of the increase in relative response rate, is larger for more disparate ratio pairs (i.e., mult FR 15 FR 60 or mult FR 30 FR 90 compared with mult FR 15 FR 30). Finally, the change in relative response rate appears to be wholly attributable to a change in absolute response rate in the component associated with the larger response requirement, since response rate in the component associated with the smaller response requirement was not systematically affected by changes in component duration.

\section{EXPERIMENT 3}

Experiment 1 showed that on a mult FR FR schedule a fourfold increase in session duration was not sufficient to produce a reliable increase in relative rate of responding for the schedule associated with the smaller response requirement. Experiment 2 showed that relative rate of responding for the smaller response requirement increased as component duration increased from 2 sec to $15 \mathrm{~min}$ and that this increase could be obtained with session durations that ranged from 30 to 90 min. In Experiment 3, pigeons were given continuous access to a mult FR FR schedule (i.e., 23.5-h experimental sessions). This procedure is an extension of that studied in Experiments 1 and 2. First, continuous access constitutes the logical limit to increasing session duration; if longer sessions increase relative rate of responding for the smaller response requirement, responding on the larger ratio schedule should be poorly maintained. More importantly, longer experimental sessions permit an investigation of longer component durations (e.g., 23.5 h).

\section{Method}

Sabjects. Two adult male White Carneaux pigeons were maintained only by the reinforcements delivered during the experimental sessions. Pigeon 37 was experimentally naive. Pigeon 82 had been employed in several previous experiments.

Apparatus. The apparatus consisted of two experimental chambers identical to the one described for Experiment 1, except that cups were added to provide free access to grit and water.

Procedure. The reinforcement schedule was mult FR X FR Y $(X<Y)$. The FR $X$ schedule was associated with a blue key light, and the FR Y schedule, with a white key light. For Pigeon 37, $X=24, Y=72$; for Pigeon 82, $X=40, Y=120$.

Component duration was varied between $30 \mathrm{sec}$ and $23.5 \mathrm{~h}$. The pigeons had continuous access to the mult schedule except from 10:30 a.m. to 11:00 a.m. each day. This time was used for maintenance of the experimental chambers.

An experimental session was defined as the 23.5-h period during which the pigeons had continuous access to the mult schedule,
Table 3

The Sequence of Experimental Conditions and Number of Sessions for Experiment 3

\begin{tabular}{|c|c|c|c|c|c|}
\hline \multicolumn{3}{|c|}{$\begin{array}{c}\text { Subject } 37 \\
\text { MULT FR 24 FR } 72\end{array}$} & \multicolumn{3}{|c|}{$\begin{array}{c}\text { Subject } 82 \\
\text { MULT FR 40 FR } 120\end{array}$} \\
\hline $\mathrm{CD}$ & SD & NS & $\mathrm{CD}$ & SD & NS \\
\hline 15.0 & 23.5 & 15 & 1.0 & 23.5 & 27 \\
\hline 71.0 & 23.5 & 30 & 47.0 & 23.5 & 18 \\
\hline 141.0 & 23.5 & 33 & 88.0 & 23.5 & 21 \\
\hline 352.0 & 23.5 & 24 & 352.0 & 23.5 & 15 \\
\hline 1410.0 & 47.0 & 21 & 1410.0 & 47.0 & 15 \\
\hline 5.0 & 23.5 & 30 & 5.0 & 23.5 & 21 \\
\hline 1.0 & 23.5 & 21 & .5 & 23.5 & 30 \\
\hline .5 & 23.5 & 21 & 705.0 & 23.5 & 24 \\
\hline 705.0 & 23.5 & 24 & & & \\
\hline
\end{tabular}

Note-CD = component duration (in minutes); $S D=$ session duration (in hours); NS = number of sessions.

except that when each component was $23.5 \mathrm{~h}$ long, a session was defined as the 48-h period required to complete one cycle of the mult schedule. The sequence of experimental conditions for each pigeon is given in the order shown in Table 3. All other procedural details were the same as those described for Experiment 2.

\section{Results}

The values in Figures 4 and 5 are the means of data collected in nine consecutive sessions that met the stability criterion. Figure 4 shows relative response rate as a function of component duration for each pigeon. For Pigeon 37, component duration had little effect on relative response rate up to a component duration of $23.5 \mathrm{~h}$, at which point relative response rate decreased to 0.55 . At all other component durations, relative response rate was greater than $\mathbf{0 . 9 0}$. For Pigeon 82, relative response rate increased as component duration increased between $30 \mathrm{sec}$ and 47 min. Relative response rate decreased as component duration increased between $47 \mathrm{~min}$ and $23.5 \mathrm{~h}$.

Figure 5 shows absolute response rate as a function of component duration. A comparison of Figures 4 and 5 reveals that changes in relative response rate for both birds were due primarily to changes in response rate in the component associated with the larger ratio requirement.

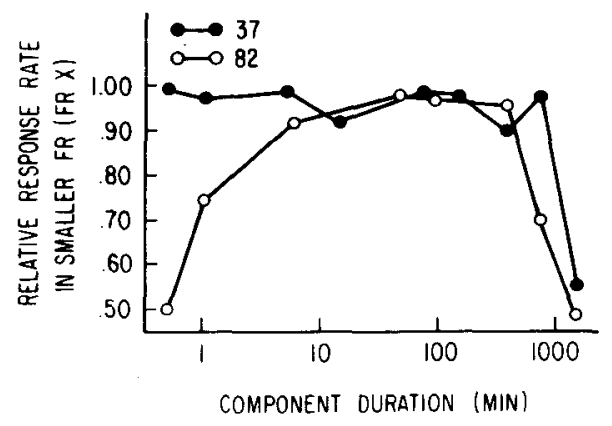

Figure 4. Relative response rate computed for smaller fixed ratio (FR X) as a function of component duration in Experiment 3 . 


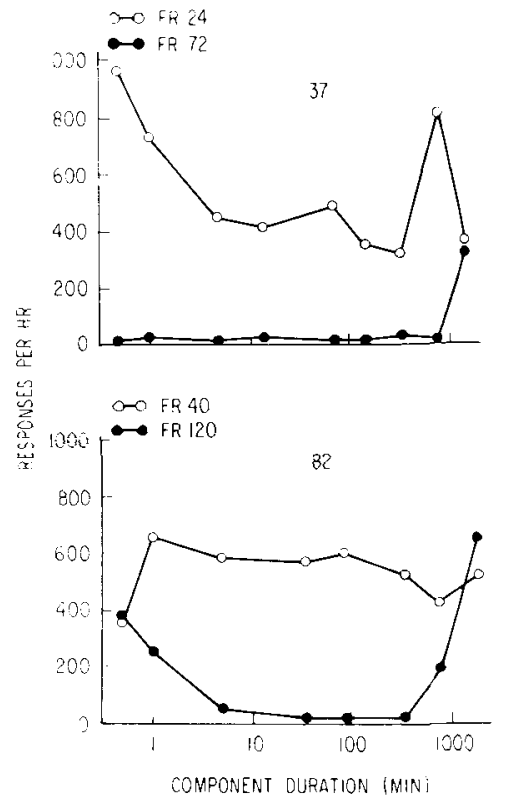

Figure 5. Responses per hour for smaller tixed ratio (FR X) and larger fixed ratlo (FR $Y$ ) as a function of component duration in Experiment 3.

\section{Discussion}

Experiment 3 confirmed and extended the results of Experiments 1 and 2. One pigeon (37) tended toward exclusive preference for the smaller ratio schedule, even at short component durations, as had Pigeon 5 in Experiment 2. For the remaining six pigeons in the two experiments, relative response rate on mult FR FR schedules was an increasing function of component durations less than $15 \mathrm{~min}$ in length. Furthermore, changes in relative response rate are due primarily to changes in the absolute response rate maintained by the larger ratio schedule and not to changes in the absolute response rate maintained by the smaller ratio schedule. Finally, the data for both Pigeon 37 and Pigeon 82 show that very long component durations produce large decreases in relative response rate.

The data in Figure 5 suggest that the overall response rate, summed over both components was a "U-shaped" function of component duration. Figure 6 (top) plots this relation directly for all three experiments. To make the contribution of each subject's data to this graph comparable, the number of responses per session was standardized within subject and within session duration to a mean of 50 and a standard deviation of 10 . The median number of responses per session in both components is plotted across log component duration (in minutes) for all three experiments. Symbols represent the number of observations used to compute the median. Here, too, a U-shaped function is evident. Final analysis of the data reveals that the shape of the function is dependent largely upon changes in the rates of responding during the FR $Y$ component as a function of duration, that is, the component associated with the larger FR requirement. Specifically, response rates were lowest at intermediate component durations during the larger FR (Figure 6, bottom).

\section{GENERAL DISCUSSION}

In addition to providing parametric data on the effect of component duration on responding in mult FR FR schedules, the present results are relevant to two important questions:

(1) Does the manipulation of a common independent variable (component duration) have similar effects on the behavior maintained by mult schedules whether the component schedules are VI or FR?

(2) If not, how does this result bear on theories that attempt to integrate data across different experimental procedures and schedule combinations?

The first question may be answered by comparing the data of Shimp and Wheatley (1971) and Todorov (1972) on the effect of component duration on responding in mult VI VI schedules with data from the present figures (especially Figure 3). Table 3 from de Villiers (1977, p. 271), which summarizes the data from the earlier experiments, shows that the response rate in the component associated with the higher rate of reinforcement is a decreasing function of component duration on a mult VI VI. In the present mult FR FR, however, response rate in the component associated with the higher rate of reinforcement is relatively invariant. On the other hand, on the mult VI VIs, response rate in the component associated with the lower rate of reinforcement is relatively invariant; in the mult FR FR, however, response rate in the component associated with the lower rate of reinforcement is a decreasing function of component duration. A necessary consequence of these functional relations (between absolute response rates and component duration) is that on a mult VI VI schedule relative response rate is a decreasing function of component duration, whereas on a mult FR FR schedule relative response rate is an increasing function of component duration. Hence, the answer to question 1 is negative; that is, the same independent variable, component duration, does not produce analogous effects on mult VI VI schedules and mult FR FR schedules.

The differences in the effect of component duration on mult VI VI schedules when compared with the effect of component duration on mult FR FR schedules suggests that a successful theoretical account of the behavior maintained by mult schedules either will be schedule specific (e.g., applicable to only certain schedule combinations) or will make differential predictions for different schedule combina- 
tions. That at least one investigator has been concerned with the problem of generality is shown by the comment of Shimp on his own formulation: "The difficulty for the present theory is that the function relating value, $V\left(E_{i}\right)$, to reinforcement delay, $D_{i}$, will not be the same, for example, in variable-ratio and variable-interval schedules. At present this dissimilarity cannot be accounted for. But the problem will have to be faced if the present model is to be generalized to the full range of classical free-operant schedules of reinforcement" (Shimp, 1969, p. 119). The present results indicate that a similar problem exists for the formulations of Catania (1973), Herrnstein (1970), and Rachlin (1973).

Herrnstein's position states that rapid alternation of components increases the parameter $m$ ( $m$ represents the degree of interaction between components and can range from 0.0 to 1.0 ) of his fundamental equation for multiple schedules:

$$
P_{1}=\frac{k R_{1}}{R_{1}+m R_{2}+R_{0}},
$$

where $P_{1}$ is the rate of responding in one component, $R_{1}$ is the rate of reinforcement for responding in that component, $\mathbf{R}_{\mathbf{2}}$ is the rate of reinforcement for responding in the other component, $\mathbf{R}_{\mathbf{0}}$ is the rate of reinforcement for responses other than $\mathbf{P}_{1}$ or $\mathbf{P}_{\mathbf{2}}$, and $k$ is a constant; in concurrent schedules, a similar formula applies with $\mathrm{m}$ equal to 1.0 . If $\mathrm{m}$ is approximately equal to 1.0 when components alternate rapidly, then the formula for relative response rate on mult schedules is identical to that for relative response rate on concurrent schedules and predicts matching. As Herrnstein (1970) has shown, matching on different-valued ratio schedules will be disconfirmed unless the animal responds exclusively on one schedule. However, the present data show that decreasing component duration (and presumably increasing $\mathrm{m}$ ) decreases relative response rate in the direction of indifference $(0.50)$. Further, the effect of decreasing $\mathrm{m}$ reduces the size of the denominator, thus leading to a prediction of higher response rate. The present results show, however, that increasing component duration (decreasing $\mathrm{m}$ ) had no effect on response rate in the component associated with the smaller response requirement and decreased, rather than increased, response rate in the component associated with the larger response requirement. Other problems with the application of Herrnstein's theory to multiple schedules have been identified by Edmon (1978), Fantino and Logan (1979, pp. 225-226), Silberberg and Schrot (1974), and Spealman and Gollub (1974). The decrease in response rate with increases in component duration in the component associated with the larger response requirement (lower rate of reinforcement) in the present mult FR FR schedules is also inconsistent with the positions of Catania (1973) and Rachlin (1973), as Norborg (1974) has discussed in detail.

At best, current theories of free-operant behavior appear to adequately describe only data collected using a restricted range of schedule combinations. This problem may be closely related to the issues of transitivity and unidimensional control raised by Fantino and Navarick (1974) and Navarick and Fantino $(1974,1975)$. For certain experimental procedures and schedule combinations (e.g., conc VI VI and mult VI VI), it may be appropriate to assume unidimensional control of response rate by the rate of reinforcement obtained on the respective schedules. On the other hand, an analogous assumption with respect to mult FR FR schedules is more likely inappropriate.

The obvious differences between VI and FR schedules suggest a possible explanation for the results of the present study. On VI schedules, both overall rate of reinforcement and the distribution of reinforcement are relatively unaffected by the pigeon's behavior. However, both of these variables are readily affected by a pigeon's performance on an FR schedule. If we assume that a particular pattern of food intake (distribution of reinforcement) is preferred, then we can also infer that responding might maintain this preferred food-intake pattern. Such accommodation is more readily accomplished on ratio than on interval schedules. Moreover, Collier, Hirsch, and Hamlin (1972) have shown that normal food-intake patterns persist, though modified, under FR schedules (see also Collier, Hirsch, and Kanarek, 1977). The data in Figure 6 (bottom) are consistent with this possibility: At short component durations, responding on the long FR is required to maintain an optimal meal duration; at very long component durations, responding on the long FR is required to maintain normal intermeal durations; neither constraint exists at intermediate component durations. Although we have no data bearing on this point conclusively, Norborg (1974) has argued that the present data may be understood readily in terms of preserving optimal meal durations. It is also possible, however, that the difference between the present results and those from multiple VI schedules is at least partially attributable to our use of a closed economy and the use of an open economy in prior studies. Norborg (1974, Experiment 4) found no systematic relation between relative response rate and component duration in a closed economy when the component schedules were variable-ratio rather than fixed-ratio, suggesting that the effect is schedule-specific. In order to assess this possibility definitively, however, mult VI VI schedules should be studied as a function of component duration in a closed economy.

In summary, current theories of free-operant behavior appear, at best, to describe adequately only 

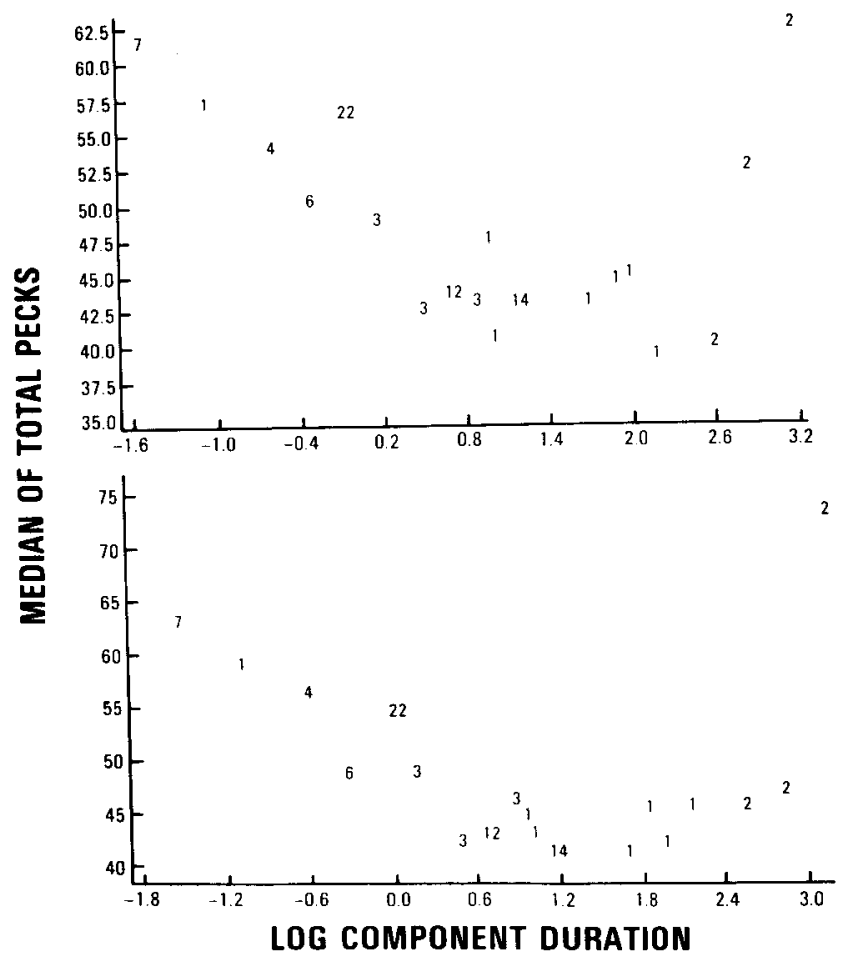

Figure 6. Median number of responses per session in both components (top) and in the component with the larger FR (bottom), each as a function of the $\log$ of component duration (in minutes) across all three experiments. Symbols denote the number of observations used to compute the median data standardized as noted in text.

data collected using a restricted range of schedule and/or deprivation combinations. This failure may be related to the lack of unidimensional control of behavior characteristic of at least certain reinforcement schedules. Lack of unidimensional control may be especially characteristic of schedules, like ratio schedules, in which parameters such as the rate and distribution of reinforcement are more directly determined by the subject's behavior.

\section{REFERENCES}

Catania, A. C. Self-inhibiting effects of reinforcement. Journal of the Experimental Analysis of Behavior, 1973, 19, 517-526.

Collier, G. H., Hirsch, E., \& Hamun, P. H. The ecological determinants of reinforcement in the rat. Physiology \& Behavior, $1972,9,705-716$.

Collien, G., Hirsch, E., \& Kanarex, R. The operant revisited. In W. K. Honig \& J. E. R. Staddon (Eds.), Handbook of operant behavior. Englewood Cliffs, N.J: Prentice-Hall, 1977.

Crossman, E. K. Pause relationships in multiple and chained fixed-ratio schedules. Journal of the Experimental Analysis of Behavior, 1968, 11, 117-126.

Crossman, E. K. The effects of fixed-ratio size in multiple and mixed fixed-ratio schedules. Psychological Record, 1971, 21, 535-544.

DE VILLIE Rs, P. A. Choice in concurrent schedules and a quantitative formulation of the law of effect. In W. K. Honig \&
J. E. R. Staddon (Eds.), Handbook of operant behavior. Englewood Cliffs, N.J: Prentice-Hall, 1977.

Edmon, E. L. Multiple schedule component duration: A reanalysis of Shimp and Wheatley (1971) and Todorov (1972). Journal of the Experimental Analysis of Behavior, 1978, 30, 239-241.

Fantino, E., \& Logan, C. A. The experimental analysis of behavior: A biological perspective. San Francisco: Freeman, 1979.

Fantino, E., \& Navarick, D. Recent developments in choice. In G. H. Bower (Ed.), The psychology of learning and motivation. New York: Academic Press, 1974.

Ferster, C. B., \& Sxinner, B. F. Schedules of reinforcement. New York: Appleton-Century-Crofts, 1957.

FindLeY, J. D. An experimental outline for building and exploring multi-operant behavior repertoires. Journal of the Experimental Analysis of Behavior, 1962, 5, 113-166.

HERRNSTEIN, R. J. On the law of effect. Journal of the Experimental Analysis of Behavior, 1970, 13, 243-266.

Hernnstein, R. J. Derivatives of matching. Psychological Review, 1979, 86, 486-495.

HURSH, S. R. Economic concepts for the analysis of behavior. Journal of the Experimental Analysis of Behavior, 1980, 34, 219-238.

LANDER, D. G., \& Irwin, R. J. Multiple schedules: Effects of the distribution of reinforcements between components on the distribution of responses between components. Journal of the Experimental Analysis of Behavior, 1968, 11, 517-524.

NAvarick, D. J., \& Fantino, E. Stochastic transitivity and unidimensional behavior theories. Psychological Review, 1974, 81, 426-441.

Navarick, D. J., \& Fantino, E. Stochastic transitivity and the unidimensional control of choice. Learning and Motivation, 1975, 6, 179-201.

Nevin, J. A., \& Shettleworth, S. J. An analysis of contrast effects in multiple schedules. Journal of the Experimental Analysis of Behavior, 1966, 9, 305-315.

Norsorg, J. Component duration and response rates on multiple ratio schedules. Unpublished doctoral dissertation, University of California, San Diego, 1974.

RAchlin, H. Contrast and matching. Psychological Review, 1973, 80, 214-234.

REYNOLDS, G. S. Some limitations on behavioral contrast and induction during successive discrimination. Journal of the Experimental Analysis of Behavior, 1963, 6, 131-139.

Schuster, C. Response latencies as a measure of the interaction of components on a multiple fixed-ratio schedule. Journal of the Experimental Analysis of Behavior, 1959, 2, 259. (Abstract)

SHIMP, C. P. Optimal behavior in free-operant experiments. Psychological Review, 1969, 76, 97-112.

ShIMP, C. P. Probabilistic discrimination learning in the pigeon. Journal of Experimental Psychology, 1973, 97, 292-304.

Shimp, C. P., \& WheAtrey, K. L. Matching to relative reinforcement frequency in multiple schedules with short component durations. Journal of the Experimental Analysis of Behavior, $1971,15,205-210$.

SilberberG, A., \& Schrot, J. A. Yoked-chamber comparison of concurrent and multiple schedules: The relationship between component duration and discrimination. Journal of the Experimental Analysis of Behavior, 1974, 22, 21-30.

Spenlman, R. D., \& Gollub, L. R. Behavioral interactions in multiple variable interval schedules. Journal of the Experimental Analysis of Behavior, 1974, 22, 471-481.

ToDorov, J. C. Component duration and relative response rates in multiple schedules. Journal of the Experimental Analysis of Behavior, 1972, 17, 45-49.

\section{NOTE}

1. Data points at the longest component durations (i.e., $7.5 \mathrm{~min}, 30-\mathrm{min}$ sessions, and $15 \mathrm{~min}, 60$ - and 90 -min session) 
were initially determined with the first component of an experimental session being the component associated with the smaller response requirement (FR X). On the second determination, the first component of an experimental session was the component associated with the larger response requirement (FR Y). The close agreement between these two determinations suggests that which component initiated an experimental session (FR $X$ or FR Y) did not contribute importantly to the results.

(Manuscript received August 4, 1981;

revision accepted for publication October 4,1982 .)

\title{
NOTICE
}

Nominations are being solicited for the editorship of Animal Learning \& Behavior. The term of the present editor, Robert C. Bolles, expires at the end of 1984. The new editor's 4-year term will begin January 1,1985 . The deadline for the receipt of nominations is April 15, 1983. It is expected that the Publications Committee of the Psychonomic Society will make the appointment by November 1983 .

Nominations (including self-nominations) should be sent to:

\author{
Robert Rescorla \\ Chair, Search Committee \\ Department of Psychology \\ University of Pennsylvania \\ 3815 Walnut Street \\ Philadelphia, Pennsylvania 19104
}

